\title{
The Asymptotic S-Matrix, Mass-Shell Anomalies and Observables ${ }^{\star}$
}

\author{
H.F. CONTOPANAGOS \\ University of Michigan, Ann Arbor, MI 48109 , USA \\ and \\ M.B. EINHORN \\ Institute for Theoretical Physics, University of California \\ Santa Barbara, CA 9\$106, USA \\ $\mathcal{B}$ \\ University of Michigan, Ann Arbor, MI 48109, USA ${ }^{\dagger}$
}

\section{Abstract}

Asymptotic S-Matrix techniques are applied to the identification of a Standard Model background to polarized deep-inelastic scattering experiments at HERA in search of Right-handed Charged Currents. It is also pointed out that the Asymptotic S-Matrix produces finite observables free of Mass-Shell Anomalies.

\section{Introduction}

Experiments using polarized beams are important for testing various aspects of the Standard Model (SM). One of these aspects is the existence of left-handed charged currents, but the absence of right-handed charged currents. One of the goals of experimentation at HERA will be to test this hypothesis by means of Deep Inelastic Scattering (DIS) experiments using a right-handed polarized electron beam. ${ }^{[1][2]}$ If such an experiment yields a signal of the type $e_{R}^{-}+p \rightarrow$ $X+$ missing energy, one may be led to the conclusion that the signal is due to a right-handed coupling of the form $g_{R} \bar{\Psi}_{e} \gamma_{\mu}\left(1+\gamma_{5}\right) \Psi_{\nu} W_{R}^{(-)_{\mu}}+$ h.c. In what follows we shall show that this connection should not be immediately made, since the SM left-handed charged current provides a finite background under the given experimental conditions at HERA.

\section{Right-handed Charged Current}

Before taking up the discussion of the background, let us first record the cross section for a right-handed coupling between a neutrino, electron and charged vector boson $W_{R}$. In what follows we shall assume that the corresponding coupling constant is the same as the Standard Model coupling constant for the left-handed coupling. We shall denote the unknown mass of the new vector boson by $M_{R}$. The neutrino is assumed massless. Then the dominant process is shown in Fig.

* Talk presented by H. F. Contopanagos.

$\dagger$ Permanent address. 
1a, where kinematic notation is also introduced. The standard kinematic variables are $s=(p+l)^{2}, \quad q^{2}=\left(l-l^{\prime}\right)^{2} \equiv-Q^{2}, \quad x \equiv Q^{2} / 2 p \cdot q$. The differential cross section for a right-handed helicity electron to undergo DIS producing a right-handed neutrino via the exchange of $W_{R}$ is

$$
\frac{d \sigma_{R}^{R}}{d x}=\frac{\pi \alpha_{2}^{2}}{2 M_{R}^{2}}\left[\left(1+\frac{1}{x \zeta_{R}}\right)^{-1} U(x)+\left(1+\frac{2}{x \zeta_{R}}-\frac{2}{x \zeta_{R}}\left(1+\frac{1}{x \zeta_{R}}\right) \ln \left(1+x \zeta_{R}\right) \bar{D}(x)\right)\right]
$$

In the above, $U(\bar{D})$ denotes the sum of contributions from quarks (antiquarks) of charge $+2 / 3(+1 / 3)$, where the $Q^{2}$-evolution has been ignored. ${ }^{\star}$ We have also used the notation $\alpha_{2} \equiv g_{2}^{2} / 4 \pi$, with $g_{2}=e / \sin \theta_{w}$, and $\zeta_{R}=s / M_{R}^{2}$. The above formula shows that an upper limit on $\sigma_{R}^{R}$ may be interpreted as a lower limit on $M_{R}$. Estimates have suggested ${ }^{[3]}$ that, in the case of a light, Dirac $\nu_{e}$ such as the one we are using here, experiments at HERA might be sensitive to $M_{R}$ as large as $300-500 \mathrm{GeV}$.

\section{The HERA Background}

This background consists of the process ${ }^{[t]} e_{R}^{-}+p \rightarrow \nu_{e L}+\gamma+X$. In other words, a right-handed helicity electron flips its helicity, while radiating an unobservable forward-going photon, and couples to a SM left-handed charged current, see Fig. 1b. The collinear photon goes unobservably down the beam pipe within a forward angle whose size is restricted by the HERA luminosity monitors.

One might think that the cross-section resulting from the amplitude in Fig. $1 b$ is heavily suppressed, not so much due to the extra coupling $\alpha$ relative to the non-radiative process, but primarily due to the helicity-flip factor $\chi_{e}^{2} \equiv$ $\left(m_{e} / E\right)^{2} \simeq 4.0 \times 10^{-10}$ (the electron energy $E$ at HERA is $\simeq 26 \mathrm{GeV}$ ). However, upon integration over the forward direction, a cancellation of the helicity-flip factor occurs, due to the collinear singularity of the electron propagator. ${ }^{[5]}$ With the kinematics shown in Fig. 1b, the cross-section can be written

$$
d \sigma_{\mathcal{L}}^{R, \gamma}=\frac{\alpha \alpha_{2}^{2}}{16 \pi^{2}} \int d^{4} q \int \frac{d \Omega_{\gamma} k_{0}^{2}}{k \cdot(l-q)} \frac{m_{e}^{2}}{(k \cdot l)^{2}}\left(\frac{p \cdot k}{p \cdot l}\right)^{2} \frac{1}{y^{\prime}}\left[x y^{\prime 2} G_{1}+\left(1-y^{\prime}\right) G_{2}+x y^{\prime}\left(1-\frac{y^{\prime}}{2}\right) G_{3}\right]
$$

where $y^{\prime}=p \cdot q / p \cdot(l-k)$. Integrating over the photon direction inside a forward cone around $l$, defined by the angular resolution $\Delta_{\theta}^{f}$ of the final state in the actual experimental situation, we have:

$$
\frac{k_{0}^{2} m_{e}^{2}}{4 \pi} \int \frac{d \Omega_{\gamma}}{(k . l)^{2}} \simeq \chi_{e}^{2} \int_{0}^{\Delta_{\theta}^{f}} \frac{d \theta^{2}}{\left(\theta^{2}+\chi_{e}^{2}\right)^{2}}=\chi_{e}^{2}\left[\frac{1}{\chi_{e}^{2}}-\frac{1}{\chi_{e}^{2}+\left(\Delta_{\theta}^{f}\right)^{2}}\right]
$$

At HERA $\Delta_{\theta}^{f} \simeq 10^{-3} ; \chi_{e} \simeq 2.0 \times 10^{-5}$. Therefore the above factor is approx-

* In other words the usual structure functions are given in terms of quark distributions by $G_{2}=2 x G_{1}=2 x(U(x)+\bar{D}(x)), \quad G_{3}=2(U(x)-\bar{D}(x))$. 
imately equal to $1 !^{\dagger}$ Inputing the quark-parton model distributions, as before, we obtain:

$$
\begin{aligned}
\frac{d \sigma_{L}^{R_{1} \gamma}}{d x}= & \frac{\alpha \alpha_{2}^{2}}{4 x s}\left\{\left[\frac{x \zeta_{L}}{2}+1-\left(1+\frac{1}{x \zeta_{L}}\right) \ln \left(1+x \zeta_{L}\right)\right] U(x)\right. \\
& \left.+\left[\frac{x \zeta_{L}}{2}-6+4\left(1+\frac{1}{x \zeta_{L}}\right) \ln \left(1+x \zeta_{L}\right)+2\left(1-\frac{1}{x \zeta_{L}}\right) \operatorname{Li}_{2}\left(x \zeta_{L}\right)\right] \bar{D}(x)\right\}
\end{aligned}
$$

where $\mathrm{Li}_{2}$ is the dilogarithm and $\zeta_{L} \equiv s / M_{W}^{2}$. This cross-section corresponds to a considerable background, as can be seen in Fig. 2.

\section{Theoretical problems and the Asymptotic S-Matrix}

The fact that the above process, calculated at an electron energy very high relative to $m_{e}$, gives a non-zero contribution identical to the massless limit of the process, makes the prediction suspicious. ${ }^{[s]}$ Lee and Nauenberg's conclusion that the helicity-flip cross-section survives in the limit $m_{e} \rightarrow 0$ is profoundly troublesome since, if taken seriously via such arguments as the calculation of section 3 , it would correspond to a mass-singularity-induced anomaly (massshell anomaly). In other words, a Lagrangian (such as $\mathcal{L}_{Q E D}$ ) that is chirally invariant in the massless limit produces chiral symmetry breaking effects, such as the non-decoupling of the electron-helicity states for certain processes, in the massless limit. For a complete review of the problem, the associated difficulties having to do with different regularization schemes of the mass singularities, and its resolution, see Ref. ${ }^{[6]}$ and references contained therein.

Suffice it here to say that the important physical ingredient that becomes relevant in calculating the high-energy limit of certain processes in perturbation theory is the physical degeneracy of free-particle states with different particle content, within the experimental resolutions of the actual physical process (experiment). Consider, as an example, the Hamiltonian of Quantum Electrodynamics, $H_{Q E D}=H_{0}+V$, where $H_{0}$ is the free-particle Hamiltonian and $V$ the interaction Hamiltonian. Because of the masslessness of the photon, the asymptotic behaviour of scattering states $e^{-i H t}|\psi\rangle$ do not approach free-particle states $e^{-i H_{0} t}\left|\psi_{0}\right\rangle$ in the limit $t \rightarrow \pm \infty$. The long-range tale of the Coulomb potential survives in the remote past and far future, making the one-electron Fock state surrounded by a soft photon cloud. On the other hand, this same masslessness of the photon, allows the soft cloud to be unobservable within the detector energy resolution i.e., degenerate with the one-particle state. Omitting this effect from the usual Feynman-Dyson S-matrix $\left(S_{F D}\right)$ i.e., assuming the asymptotic Hamiltonian of QED is $H_{0}$, gives rise to IR divergencies and doesn't describe the actual physical degeneracy. In general, omitting an asymptotic interaction from the

I Notice that we would recover the same result even in the massless limit $\chi_{e} \rightarrow 0$, i.e., for an exactly massiess electron, after the forward integration. 
asymptotic Hamiltonian of a massless gauge theory produces mass-singularities, mass-shell anomalies, and disregards physical degeneracy. The high-energy limit of a massive theory, calculated via the resulting $S_{F D}$, resurrects these problematic (but complementary) features.

One can properly account for the asymptotic properties of these theories, by choosing an interacting asymptotic Hamiltonian:

$$
H_{0} \rightarrow H_{A}(\Delta)=H_{0}+V_{A}(\Delta)
$$

The physical meaning of $\Delta$ will be explained later in this section. The corresponding Asymptotic S-matrix is

$$
S_{A}=\Omega_{H, H_{A}}^{(-) \dagger} \Omega_{H, H_{A}}^{(+)}=\Omega_{H_{A}, H_{0}}^{(-)} S_{F D} \Omega_{H_{A}, H_{0}}^{(+) \dagger}
$$

with the Møller wave operators defined as $\Omega_{H, H_{A}}^{(\mp)} \equiv \lim _{t \rightarrow \pm \infty} e^{i H t} e^{-i H_{A} t}=$ $\Omega_{H, H_{0}}^{(\mp)} \Omega_{H_{\mathcal{A}}, H_{0}}^{(\mp)}$. Transforming all operators in the interaction picture we may write:

$$
\Omega_{H_{A}, H_{0}}^{( \pm)}=T \exp \left[-i \int_{\mp \infty}^{0} d t V_{A}(t)\right], \quad S_{F D}=T \exp \left[-i \int_{-\infty}^{+\infty} d t V(t)\right]
$$

In this picture a perturbative evaluation of $S_{A}$ is straightforward. Suppose we are interested in QED radiative corrections to a basic process that occurs in a theory with an interaction Hamiltonian $V(t)$. One can write $V(t)=V^{(Q E D)}(t)+V^{(J)}(t)$, where $V^{(J)}(t)$ is the rest of the Hamiltonian, giving rise to the non-radiative process (such as the one in section 2). Then:

$$
V^{(Q E D)}(t)=e \int d^{3} x: \bar{\Psi}^{(e)} \gamma_{\mu} \Psi^{(e)}: A^{\mu}=e \int \widehat{d^{3} k_{1}} \widehat{d^{3} k_{2}} \sum_{l=1}^{8} h_{l}\left(\mathbf{k}_{1}, \mathbf{k}_{2}, \mathbf{k}_{3}\right) \exp \left[-i(S \omega)^{l} t\right]
$$

In the above expression $\mathcal{S}$ is a $8 \times 3$ sign matrix and $\omega$ stands for the energies of the particles in each vertex $h_{l}$. We can now define the asymptotic interaction Hamiltonian as

$$
V_{A}^{(Q E D)}(\Delta ; t)=e \int \widehat{d^{3} k_{1}} \widehat{d^{3} k_{2}} \sum_{l=1}^{8} h_{l}\left(\mathbf{k}_{1}, \mathbf{k}_{2}, \mathbf{k}_{3}\right) \Theta\left(\Delta-\left|(\mathcal{S} \omega)^{l}\right|\right) \exp \left[-i(\mathcal{S} \omega)^{l} t\right]
$$

We see that $\Delta$ corresponds to the experimental regions of phase space characterizing a certain physical process within which the energies $(\mathcal{S} \omega)^{l}$ of the particles at the corresponding vertex $l$ are indistinguishable (degenerate). More precisely, one may write:

$$
\Delta=U \Delta_{\alpha}, \Delta_{\alpha} \in\left\{\delta_{\theta}^{i n}, \Delta_{\theta}^{f}, \delta_{E} \ldots\right\}
$$

where $\delta_{\theta}^{i n}=$ the beam angular resolution, $\Delta_{\theta}^{f}=$ the final-state angular resolution, $\delta_{E}=\Delta E / E, \Delta E=\max \left\{\Delta E^{i n}, \Delta E^{f}\right\}=$ the energy resolution. It is obvious that 


$$
V_{A}^{(Q E D)} \begin{cases}\neq 0, & \text { if } \exists \alpha, i: \chi_{i} \equiv m_{i} / \omega_{i}<\Delta_{\alpha} \\ =0, & \text { otherwise. }\end{cases}
$$

This construction defines the high-energy limit of a theory and automatically includes the massless limit (massless theory). A perturbative evaluation of the asymptotic wave operators may be obtained from the following formula:

$$
\Omega_{ \pm}^{(n)}(\Delta)=\sum_{l_{1} l_{2} \ldots l_{n}} e^{n} V_{l_{n}} V_{l_{n-1}} \ldots V_{l_{1}} \frac{1}{(S \omega)^{l_{1}} \pm i \epsilon} \frac{1}{(\mathcal{S} \omega)^{l_{1}}+(\mathcal{S} \omega)^{l_{2}} \pm 2 i \epsilon} \ldots \frac{1}{(\mathcal{S} \omega)^{l_{1}}+\ldots(S \omega)^{l_{n}} \pm n i \epsilon}
$$

with $V_{l_{j}} \equiv \int \widehat{d^{3} k_{1_{j}}} \widehat{d^{3} k_{2 j}} h_{l_{j}}\left(\mathbf{k}_{1_{j}}, \mathbf{k}_{2_{j}}, \mathbf{k}_{3_{j}}\right) \Theta_{\Delta}$.

\section{HERA background revisited}

Let us calculate again the radiative process of section 3 , using the correct matrix $S_{A}$. Our choice of initial and final states will be:

$$
|i\rangle=|e(\mathbf{l} ; R)\rangle, \quad|f\rangle=\left|\nu_{e}\left(\mathbf{l}^{\prime} ; L\right) \gamma(\mathbf{k} ; \lambda)\right\rangle
$$

Considering the hadronic part of the process as an external source of $W$-bosons we may write:

$$
\left[S_{A}^{(1)}\right]_{f i}=\left\langle f\left|S_{F D}^{(0)} \Omega_{+}^{(1) \dagger}\right| i\right\rangle+\left\langle f\left|S_{F D}^{(1)}\right| i\right\rangle
$$

The first term is the extra contribution coming from the degeneracy of the initial state within the experimental angular resolution of the electron beam. Indeed one may show

$$
\Omega_{+}^{(1) \dagger}|i\rangle=e \sum_{\alpha \lambda^{\prime}} \int \widetilde{d^{3} k^{\prime}}\left|\gamma\left(\mathbf{k}^{\prime} ; \lambda^{\prime}\right) e\left(1-\mathbf{k}^{\prime} ; \alpha\right)\right\rangle \bar{U}_{\alpha}^{(e)}\left(\mathbf{1}-\mathbf{k}^{\prime}\right) d_{\lambda^{\prime}}\left(\mathbf{k}^{\prime}\right) U_{i}^{(e)}(\mathbf{1}) \frac{\Theta\left(\Delta-\left|\nu^{\prime}\right|\right)}{2 \omega\left(1-\mathbf{k}^{\prime}, m_{e}\right) \nu^{\prime}}
$$

In the above $\nu^{\prime}=\omega\left(\mathbf{l}-\mathbf{k}^{\prime}, m_{e}\right)+\omega\left(\mathbf{k}^{\prime}, m_{\gamma}\right)-\omega\left(\mathbf{l}, m_{e}\right)$. Notice how the asymptotic wave operator transforms a one-particle state into an electron-photon state degenerate with that one. The second term in Eq. (11) can be calculated from the Feynman rules. The $S_{A}$-matrix element is shown in Fig. 3. Looking at the collinear phase space where the final photon is almost parellel to the initial electron, we deduce the following singularity structure for the cross-section:

$$
d \sigma_{L}^{R, \gamma} \sim \alpha \chi_{e}^{2}\left\{\frac{1}{\chi_{e}^{2}+\left(\delta_{\theta}^{i n}\right)^{2}}-\frac{1}{\chi_{e}^{2}+\left(\Delta_{\theta}^{f}\right)^{2}}\right\}
$$

i) Massless limit:

Obviously $\lim _{\chi_{e}^{2} \rightarrow 0} d \sigma_{L}^{R, \gamma}=0$. Therefore there are no mass-shell anomalies in the massless limit, if one computes the physically relevant $S_{A}$-matrix elements.

ii) High-energy limit: 
In eq. (13) we shall have to input the experimental values of the physical resolutions. At HERA, $\chi_{e} \simeq 2.0 \times 10^{-5}, \Delta_{\theta}^{f} \simeq 10^{-3}$. For the inital (beam) angular resolution an adequate estimate can be given by a lower limit to the ability of the accelerator to distinguish a single-electron from a collinear electron-photon state going through the interaction region. Hence an upper limit is $\delta_{\theta}^{i n}<\sigma / d^{\star}$ where $\sigma$ is the transverse radius of the beam at the interaction region and $d$ the drift distance of the electrons from the final focus to the interaction region. At HERA, $\sigma / d \simeq 0.07 \mathrm{~mm} / 5.5 \mathrm{~m} \simeq 1.2 \times 10^{-5}{ }^{\dagger}$ Notice that in all cases we may write

$$
\chi_{e}^{2}+\left(\Delta_{\theta}^{f}\right)^{2} \simeq\left(\Delta_{\theta}^{f}\right)^{2}, \quad \chi_{e}^{2}+\left(\delta_{\theta}^{i n}\right)^{2} \simeq \chi_{e}^{2}
$$

Therefore $\lim _{\text {exp }} d \sigma_{L}^{R, \gamma} \sim \alpha$, and the prediction of the background made in section 3 is approximately correct. However, survival of this background is not connected to mass-shell anomalies any more since, as we showed, the massless limit of the process is indeed smooth and equal to zero.

\section{Conclusion}

Perturbative calculations using $S_{F D}$ in massless gauge theories or in massive theories when the high-energy limit of a physical process is sought, are plagued by mass singularities, mass-shell anomalies, and are based on matrix elements that do not account for the physical degeneracy occuring in these cases. $S_{A}$, on the other hand, incorporates in its definition the notion of physical degeneracy and is characterized by finite and non-anomalous matrix elements. Hence it allows reliable perturbative calculations in massless gauge theories or when highenergy-limit processes are considered. Processes that involve exactly massless particles, and that are anomalous when calculated via $S_{F D}$, turn out to have a smooth massless limit equal to zero when calculated via $S_{A}$. This is due to the fact that the experimental resolutions introduced by the transformation of Fock states into coherent states cut-off the mass singularities in that case. For this reason, a discussion analogous to the helicity-flip process shows that there are no longitudinal massless photons in massless QED, contrary to some recent claims. ${ }^{[]}$Processes that involve massive particles, but whose energy is much larger than their mass, may be calculated via $S_{A}$ as well. These processes, if

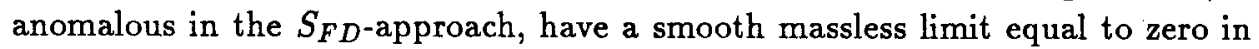
the $S_{A}$-approach, but their high-energy (experimental) limit may be non-zero, if the experimental resolutions happen to be smaller than the corresponding mass-parameters. This is the criterion defining when the high-energy limit of a process corresponds to a massive or a massless theory. Through this approach, a SM background is identified and will have to be taken into account for the polarized DIS experiments designed at HERA.

\footnotetext{
* Actually we suspect that in reality $\delta_{\theta}^{\text {in }} \ll \sigma / d$.

$\dagger$ By comparison, at SLC $\sigma / d \simeq 10^{-6}$.
} 


\section{Acknowledgements}

We are grateful to D.N. Williams, D.R.T. Jones, A. Mueller, G. Sterman, and A. Wightman for many helpful discussions at various stages of this work.

\section{Figure Captions}

Fig. 1

a. Deep-inelastic scattering via a hypothetical $W_{R}$ exchange.

b. Radiative deep-inelastic scattering via a Standard Model $W$ exchange. Fig. 2

The ratio of the signal over the background as a function of $M_{R}$.

Fig. 3

a. The two-particle state contribution to the $S_{A}$-matrix element.

b. The one-particle state contribution, corresponding to the $S_{F D}$-matrix element.

\section{REFERENCES}

1. Proceedings of the Workshop Experimentation at HERA, NIKHEF, Amsterdam, June 9-11, 1983, DESY HERA 83/20, Oct. 1983.

2. Proceedings of the HERA Workshop, Hamburg, Germany, Oct. 12-14, 1987 (R.D.Peccei, ed.) Hamburg: Deutsches Elektronen Synchrotron, 1988, 2 vols.

3. G. Wolf, Lectures given at the Advanced Study Institute on Techniques and Concepts of High Energy Physics, 1986, St. Croix, DESY 86-089, August 1986.

4. H.F. Contopanagos and M.B. Einhorn, UM-TH-89-09 (July, 1989).

5. T.D. Lee and M. Nauenberg, Phys. Rev. 133 (1964) B1549.

6. H.F. Contopanagos, Nucl. Phys. B343 (1990) 571.

H.F. Contopanagos and M.B. Einhorn, UM-TH-90-11; NSF-ITP-90-165i.

H.F. Contopanagos and M.B. Einhorn, UM-TH-90-12; NSF-ITP-90-166i.

7. A.S. Gorsky, B.L. Ioffe, and A.Yu. Khodjamirian, Phys. Lett. 227 (1989) 474 . 
304 The Asymptotic S Matrix
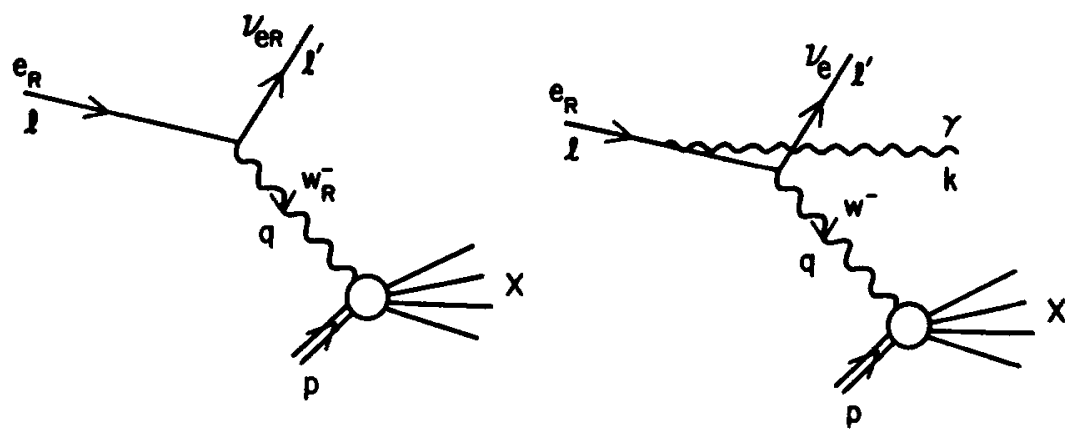

Fig. 1 a,b

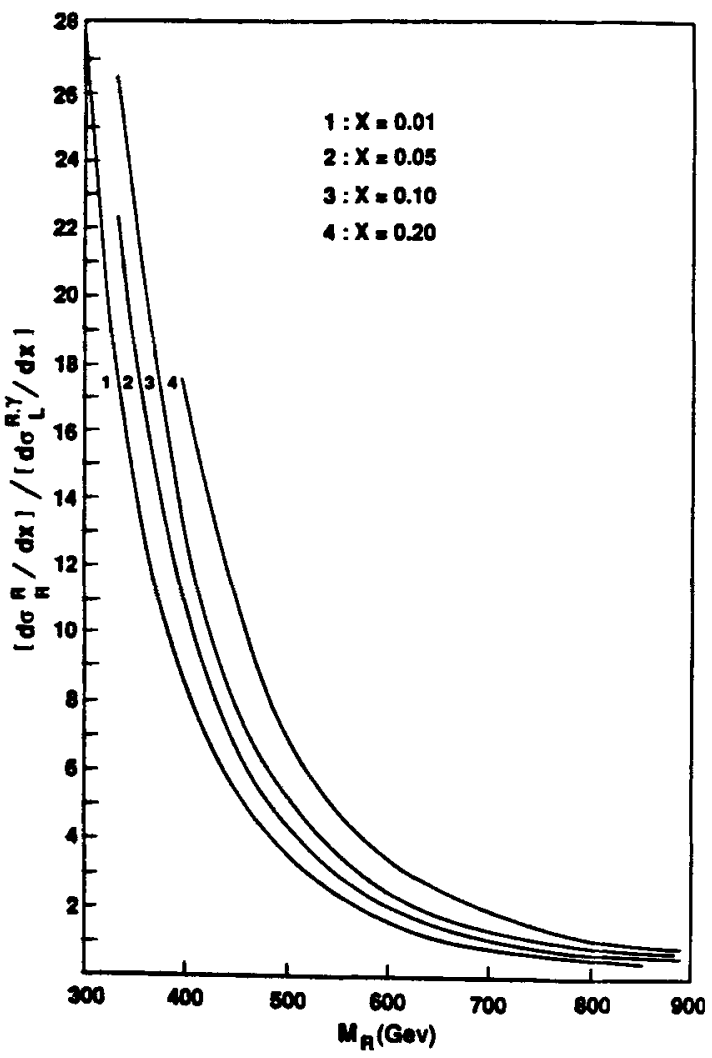

Fig. 2
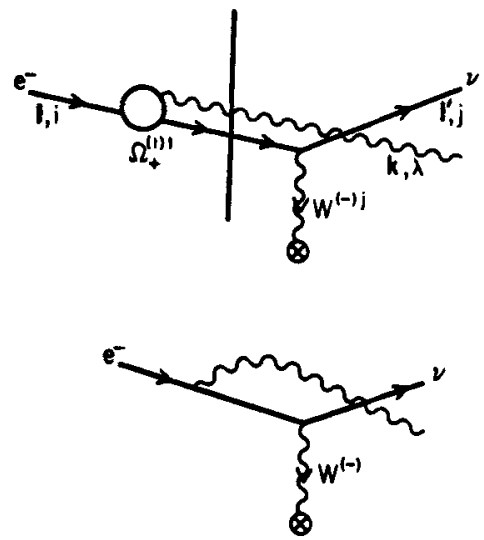

Fig 3 a,b 